\title{
Meromorphic solutions of a third order nonlinear differential equation
}

\author{
Robert Conte ${ }^{1,2, a)}$ and Tuen-Wai Ng (吳端偉 $)^{1, b)}$ \\ ${ }^{1}$ Department of Mathematics, The University of Hong Kong, Pokfulam Road, Hong Kong \\ ${ }^{2}$ LRC MESO, Centre de mathématiques et de leurs applications (UMR 8536) et \\ CEA-DAM, École normale supérieure de Cachan, 61, avenue du Président Wilson, \\ F-94235 Cachan Cedex, France
}

(Received 20 November 2009; accepted 22 January 2010; published online 26 March 2010)

\begin{abstract}
We prove that all the meromorphic solutions of the nonlinear differential equation $c_{0} u^{\prime \prime \prime}+6 u^{4}+c_{1} u^{\prime \prime}+c_{2} u u^{\prime}+c_{3} u^{3}+c_{4} u^{\prime}+c_{5} u^{2}+c_{6} u+c_{7}=0$ are elliptic or degenerate elliptic, and we build them explicitly. (C) 2010 American Institute of Physics.

[doi:10.1063/1.3319568]
\end{abstract}

\section{INTRODUCTION}

When a system is governed by an autonomous nonlinear algebraic partial differential equation (PDE), it frequently admits some permanent profile structures such as fronts, pulses, kinks, etc., ${ }^{16}$ and usually these profiles are mathematically some single-valued solutions of the traveling wave reduction $(x, t) \rightarrow x-c t$ of the PDE to an ordinary differential equation (ODE). The physical motivation of the present work is to find such solutions in closed form. Since this is a difficult mathematical problem, we restrict here to a simple case (a third order nonlinear ODE) and solve it completely. The method we used here is a refinement of Eremenko's method used in Ref. 4 as well as Refs. 5 and 6, which is based on the local singularity analysis of the meromorphic solutions of the given differential equations as well as the zero distribution and growth rate of the meromorphic solutions by using Nevanlinna theory. This is a very powerful method. For example, it was used by Eremenko ${ }^{5}$ to characterize all meromorphic traveling wave solutions of the Kuramoto-Sivashinsky (KS) equations. In fact, Eremenko showed that all the meromorphic traveling wave solutions of the KS equations belong to the class $W$ (such as Weierstrass), which consists of elliptic functions and their successive degeneracies, i.e., elliptic functions, rational functions of one exponential $\exp (k z), k \in \mathrm{C}$, and rational functions of $z$.

In general, even if we know that the solutions belong to the class $W$, it is still difficult to find their explicit form. To overcome this problem, we shall apply the subequation method introduced in Ref. 13 and developed in Ref. 3. In order to emphasize the method, we will choose a test equation according to the following criteria:

(1) to have a small differential order $n$;

(2) to have only nonrational Fuchs indices, apart from the ever present -1 index;

(3) to be of the form $u^{(n)}=P\left(u^{(n-1)}, \ldots, u^{\prime}, u\right)$, with $P$ as a polynomial of its arguments;

(4) to have movable poles of the order 1; and

(5) to be complete in the classical sense ${ }^{15}$ (see details in Ref. 2, p. 122), i.e., to include all admissible nondominant terms.

The requirement for nonrational Fuchs indices sets $n \geq 3$. Let us take the complete autonomous third order polynomial ODE with simple poles,

\footnotetext{
a)Electronic mail: robert.conte@cea.fr.

${ }^{b)}$ Electronic mail: ntw@maths.hku.hk.
} 


$$
d_{0} u^{\prime \prime \prime}+d_{1} u u^{\prime \prime}+d_{2} u^{\prime 2}+d_{3} u^{2} u^{\prime}+d_{4} u^{4}+c_{1} u^{\prime \prime}+c_{2} u u^{\prime}+c_{3} u^{3}+c_{4} u^{\prime}+c_{5} u^{2}+c_{6} u+c_{7}=0 .
$$

This equation is indeed complete in the sense that it includes all polynomial terms having a singularity degree at most equal to 4 , as seen from the generating function

$$
\begin{aligned}
\frac{1}{(1-t u)\left(1-t^{2} u^{\prime}\right)\left(1-t^{3} u^{\prime \prime}\right)}= & 1+u t+\left(u^{2}+u^{\prime}\right) t^{2}+\left(u^{3}+u u^{\prime}+u^{\prime \prime}\right) t^{3} \\
& +\left(u^{4}+u^{2} u^{\prime}+u^{\prime 2}+u u^{\prime \prime}\right) t^{4}+O\left(t^{5}\right) .
\end{aligned}
$$

Let us choose one particular set of dominant terms (the ones with coefficients $d_{j}$, which have quadruple poles) so as to enforce from the beginning the condition that the Fuchs indices be nonrational. After setting $c_{3}=0$, without loss of generality, our test equation will be normalized as

$$
c_{0} u^{\prime \prime \prime}+6 u^{4}+c_{1} u^{\prime \prime}+c_{2} u u^{\prime}+c_{4} u^{\prime}+c_{5} u^{2}+c_{6} u+c_{7}=0,
$$

Let $u$ be a meromorphic solution of the ODE (3). We first check that if $u$ has a movable pole at $z=z_{0}$, then $u$ has only three distinct Laurent series expansions at $z_{0}$. Note that if $z_{0}$ is a pole of $u$, it must be a simple pole. Therefore, in a neighborhood of $z=z_{0}$, the Laurent series of the meromorphic solution $u$ is of the form

$$
u(z)=u_{-1}\left(z-z_{0}\right)^{-1}+u_{0}+u_{1}\left(z-z_{0}\right)+\cdots, \quad u_{-1} \neq 0 .
$$

Denote $a$ any one of the cubic roots of $c_{0}$. Substituting the above Laurent series into the ODE (3) and balancing the leading terms, we obtain $u_{-1}=a$, and $u_{0}=\left(-2 c_{1} a+c_{2} a^{2}\right) /\left(24 c_{0}\right)$. We are going to prove that there are at most three distinct Laurent series expansions at $z_{0}$. If one linearizes the ODE (3) around the movable singularity $z=z_{0}$ (Ref. 2, p. 114), the resulting linear ODE has the Fuchsian type at $z_{0}$, and its three Fuchs indices $r$ are defined by

$$
(r+1)\left(r^{2}-7 r+18\right)=0 .
$$

Hence, the Fuchs indices are equal to $r=-1,(7 \pm \sqrt{-23}) / 2$. Because of the absence of any positive integer in the set of values of $r$, all other coefficients $u_{i}$ are uniquely determined (Ref. 2, p. 90) by the leading coefficient $u_{-1}$. Hence, there are at most three meromorphic functions with poles at $z=z_{0}$ satisfying the ODE (3).

We shall study the third order nonlinear differential equation (3) and show that all meromorphic solutions of this differential equation belong to the class $W$. More specifically, our results are the following.

Theorem 1: If the ODE (3) has a particular meromorphic solution $u$, then $u$ belongs to the class W. Moreover, a necessary and sufficient condition for the ODE (3) to admit a particular meromorphic solution is to belong to the following list:

$$
\begin{gathered}
S_{3 a}: \quad c_{1}, c_{6}=\text { arbitrary, } \quad c_{2}=0, \quad c_{5}=0, \quad c_{7}=0, \quad c_{4}=\frac{c_{1}^{2}}{12 c_{0}}, \\
S_{3 b}: \quad c_{5}, c_{6}=\text { arbitrary, } \quad c_{1}=0, \quad c_{2}=0, \quad c_{4}=0, \quad c_{7}=\frac{c_{5}^{2}}{128}, \\
S_{2 A}: \quad c_{1}, c_{4}=\text { arbitrary, } \quad c_{2}=0, \quad c_{5}=\frac{c_{1}^{2}-12 a^{3} c_{4}}{4 a^{4}}, \quad c_{6}=-\frac{c_{1}\left(c_{1}^{2}+36 a^{3} c_{4}\right)}{144 a^{6}}, \\
c_{7}=\frac{\left(12 a^{3} c_{4}-c_{1}^{2}\right)\left(36 a^{3} c_{4}-11 c_{1}^{2}\right)}{1536 a^{8}},
\end{gathered}
$$




$$
\begin{gathered}
S_{2 B}: c_{1}, c_{2}=\text { arbitrary, } \quad c_{4}=\frac{44 c_{1}^{2}+8 a c_{1} c_{2}-a^{2} c_{2}^{2}}{144 a^{3}}, \\
c_{5}=\frac{-32 c_{1}^{2}-24 a c_{1} c_{2}-7 a^{2} c_{2}^{2}}{48 a^{4}}, \quad c_{6}=-\frac{\left(c_{1}+a c_{2}\right)\left(12 c_{1}^{2}+6 a c_{1} c_{2}+a^{2} c_{2}^{2}\right)}{144 a^{6}}, \\
c_{7}=-\frac{\left(4 c_{1}+3 a c_{2}\right)\left(48 c_{1}^{2}+20 a c_{1} c_{2}+a^{2} c_{2}^{2}\right)}{55296 a^{7}}, \\
S_{1}: c_{1}, c_{2}, c_{4}, c_{5}=\operatorname{arbitrary}, \\
1152 a^{6} c_{6}=-56 c_{1}^{3}+60 a c_{1}^{2} c_{2}-18 a^{2} c_{1} c_{2}^{2}+a^{3} c_{2}^{3}+288 a^{3} c_{1} c_{4}-144 a^{4} c_{2} c_{4}-96 a^{4} c_{1} c_{5}+48 a^{5} c_{2} c_{5}, \\
2^{13} 3^{2} a^{8} c_{7}=-176 c_{1}^{4}+128 a c_{1}^{3} c_{2}+24 a^{2} c_{1}^{2} c_{2}^{2}-32 a^{3} c_{1} c_{2}^{3}+5 a^{4} c_{2}^{4}+2688 a^{3} c_{1}^{2} c_{4}-1536 a^{4} c_{1} c_{2} c_{4} \\
+96 a^{5} c_{2}^{2} c_{4}-6912 a^{6} c_{4}^{2}+128 a^{4} c_{1}^{2} c_{5}-512 a^{5} c_{1} c_{2} c_{5}+224 a^{6} c_{2}^{2} c_{5}+4608 a^{7} c_{4} c_{5} \\
+2304 a^{8} c_{5}^{2} .
\end{gathered}
$$

We shall apply Eremenko's method ${ }^{5}$ to prove the first part of Theorem 1. Here, we shall assume that the readers are familiar with the standard terminology and results of Nevanlinna theory. The standard references of this theory are Refs. 8, 12, and 14 (see also Ref. 5 for a quick introduction). Our argument is slightly different from that of Eremenko and it makes use of the following version of Clunie's lemma [see Ref. 12 (Lemma 2.4.2) and see also Ref. 17].

Lemma 1: Let $f$ be a transcendental meromorphic solution of

$$
f^{n} P(z, f)=Q(z, f),
$$

where $P(z, f)$ and $Q(z, f)$ are polynomials in $f$ and its derivatives with meromorphic coefficients $\left\{a_{\lambda} \mid \lambda \in I\right\}$ such that $m\left(r, a_{\lambda}\right)=S(r, f)$ for all $\lambda \in I$. If the total degree of $Q(z, f)$ as a polynomial in $f$ and its derivatives is less than or equal to $n$, then

$$
m(r, P(r, f))=S(r, f) .
$$

Now let $u$ be a function meromorphic in the complex plane that satisfies the above ODE (3). If $u$ is rational, then we are done. So suppose $u$ is a transcendental meromorphic solution of Eq. (3), then we have

$$
-6 u^{4}=c_{0} u^{\prime \prime \prime}+c_{1} u^{\prime \prime}+c_{2} u u^{\prime}+c_{4} u^{\prime}+c_{5} u^{2}+c_{6} u+c_{7},
$$

Take $f=u, P=u, n=3$, and apply Clunie's lemma (Lemma 1 ) to the above equation, we conclude that $m(r, u)=S(r, u)$, and hence $(1-o(1)) T(r, u)=N(r, u)$. We claim that $u$ must have infinitely many poles. Assume it is not the case, then $N(r, u)=O(\log r)$. Therefore, $T(r, u)=O(\log r)$, which is impossible as $u$ is transcendental.

Second, we prove that if $u$ is a transcendental meromorphic solution, then $u$ is a periodic function. Recall that there are at most three meromorphic functions with poles at $z=z_{0}$ satisfying the ODE (3). Now let $z_{j}, j=1,2,3, \ldots$ be the poles of $u(z)$, then the functions $w_{j}(z)=u\left(z+z_{j}-z_{0}\right)$ are meromorphic solutions of the ODE (3) with a pole at $z_{0}$. Thus, some of them must be equal. Consequently, $u$ is a periodic function.

Without loss of generality, we may assume that $u$ has a period of $2 \pi i$. Let $D=\{z: 0 \leq \operatorname{Im} z$ $<2 \pi\}$. If $u$ has more than three poles in $D$, then by the previous argument, we can conclude that $u$ is periodic in $D$, and hence it is indeed an elliptic function and we are done.

Now suppose $u$ has at most three poles in $D$. Since $u$ is a periodic function with period $2 \pi i$, we have $N(r, u)=O(r)$, as $r \rightarrow \infty$. It follows from $(1-o(1)) T(r, u)=N(r, u)$ that $T(r, u)=O(r)$. By 
Nevanlinna's first fundamental theorem, we know that for any $a \in \mathbb{C}, N(r, 1 /(u-a))=O(r)$ as $r$ $\rightarrow \infty$. By the periodicity of $u$, we conclude that $u$ takes each $a$ finitely many times in $D$. Hence, the function $R(z)=u(\ln z)$ is a single-valued analytic function in the punctured plane $C \backslash\{0\}$ and takes each $a \in \mathrm{C}$ finitely many times. It follows that 0 is a removable singularity of $R$ and $R$ can then be extended to a meromorphic function on C. Hence, $R$ is a rational function as it takes each complex number finitely many times. Therefore, $u(z)=R\left(e^{z}\right)$ belongs to the class $W$ and this completes the proof of the first part of Theorem 1.

Remark: From the above proof, we notice that if $u$ is an elliptic solution, then $u$ has at most three (simple) poles in each fundamental polygon $\Omega$. Recall that the residue of $u$ at any pole must be one of $a, \omega a$, and $\omega^{2} a$, where $\omega$ is the cubic root of unity. Since the sum of the residues of all the poles in any fundamental polygon $\Omega$ is zero, $u$ must have three distinct simple poles in $\Omega$, and hence we have three distinct Laurent series at $z_{0}$.

Remark: If $u(z)=R\left(e^{k z}\right)$, where $R$ is some rational function, then $R$ has at most three (simple) poles in $C \backslash\{0\}$. We are going to show that $R$ cannot have a pole at 0 . Suppose we write $u(z)$ $=R(Z)=r_{0} / Z^{n}+\sum_{i=1}^{3} r_{i} /\left(Z-Z_{i}\right)+q(Z)$, where $q$ is a polynomial in $Z=e^{k z}$. Substituting $u(z)=R(Z)$ into ODE (3) and letting $Z$ tend to infinity, we can conclude that $q$ equals to some constant $C$. Now letting $Z$ tend to 0 , we can deduce that $r_{0}=0$. Hence, $u(z)=\sum_{i=1}^{3} r_{i} /\left(e^{k z}-Z_{i}\right)+C$, where $Z_{i}, C \in \mathrm{C}$. Finally, if $u$ is rational, then $u$ will have at most three (simple) poles in C. Similarly, we can show that $u$ must be of the form $\Sigma_{i=1}^{3} r_{i} /\left(z-z_{i}\right)+C$, where $r_{i}, C \in \mathrm{C}$.

\section{EXPLICIT SOLUTIONS IN THE CLASS $W$}

Let us determine the constraints on the coefficients $c_{j}$ of (3) for meromorphic solutions to exist, and let us determine all these meromorphic solutions in closed form. According to Sec. I, these solutions are necessarily elliptic or degenerate of elliptic (i.e., rational in one exponential $e^{k z}, k \in \mathrm{C}$ or rational in $z$ ), i.e., they belong to the class $W$.

If the meromorphic solution is elliptic, by a classical theorem, the sum of the residues of the three Laurent series for $u$ [Eq. (4)] must vanish, and similarly for any rational function of $u, u^{\prime}$, and $u^{\prime \prime}$. These necessary conditions ${ }^{10}$ are first established in Sec. II A.

If the solution is elliptic, one knows the elliptic orders of $u$ and $u^{\prime}$, they are, respectively, equal to three (three simple poles) and six (three double poles). Therefore, by a classical theorem of Briot and Bouquet [see Ref. 1 (p. 277), Ref. 7 (Pt. II, Chap. IX, p. 329), and Ref. 9 (p. 424)], the elliptic solution obeys a first order algebraic equation whose degree in $u^{\prime}$ is of the order of $u$ (three) and degree in $u$ is of the order of $u^{\prime}$ (six),

$$
F\left(u, u^{\prime}\right) \equiv \sum_{k=0}^{m} \sum_{j=0}^{2 m-2 k} a_{j, k} u^{j} u^{\prime k}=0, \quad a_{0, m} \neq 0,
$$

with $m=3$. The complex constants $a_{j, k}$, with $a_{0, m} \neq 0$, are then determined by the algorithm presented in Ref. 13, i.e., by requiring each of the three Laurent series (4) to obey (12). The search for all third degree subequations (12) obeyed by the three Laurent series is performed in Sec. II B.

As to those solutions of (3), which are degenerate of elliptic, they also obey a first order equation (12), whose degree $m$ is at most 3. Because of the singularity structure of (3) (three distinct Laurent series), any $m$ th degree subequation, $1 \leq m \leq 3$, must have $m$ distinct Laurent series. The search for all second or first degree subequations (12) is performed in Secs. II C and II D.

Let us first establish all these first order subequations. Their general solution may be either single valued (and hence in class $W$ ) or multivalued. The explicit integration of the single-valued subset will provide as a final output all the meromorphic solutions of (3) in closed form.

\section{A. Residue conditions}

If (3) admits an elliptic solution, it is necessary that, for any rational function of $u$ and its derivatives, the sum of the residues inside a period parallelogram be zero, 


$$
\forall k \in \mathbb{N} \forall n \in \mathbb{N} \operatorname{res} \sum_{i=1}^{3}\left(u^{(k)}\right)^{n}=0 .
$$

The first conditions are

$$
\begin{cases}k=0, & n=2: \quad c_{2}=0 \\ k=0, & n=3: \quad c_{4}=\frac{c_{1}^{3}}{12 a^{3}} \\ k=0, & n=5: \quad c_{1} c_{5}=0 \\ k=0, & n=7: \quad c_{1} c_{7}=0 \\ k=1, & n=4: \quad\left(c_{6}\left(c_{5}^{2}-128 c_{7}\right)=0 \quad \text { if } c_{1}=0\right), \quad\left(c_{7}\left(c_{1}^{3}+36 a_{0}^{2} c_{6}\right)=0 \quad \text { if } c_{1} \neq 0\right) .\end{cases}
$$

When the computation is limited to $k \leq 4, n \leq 10$, this defines three and only three distinct sets of fixed coefficients for a possible elliptic solution,

$$
\begin{gathered}
c_{2}=0, \quad c_{1}=0, \quad c_{4}=0, \quad c_{6} \neq 0, \quad c_{7}=\frac{c_{5}^{2}}{128}, \\
c_{2}=0, \quad c_{1}=0, \quad c_{4}=0, \quad c_{6}=0, \\
c_{2}=0, \quad c_{1} \neq 0, \quad c_{4}=\frac{c_{1}^{2}}{12 a^{3}}, \quad c_{5}=0, \quad c_{7}=0 .
\end{gathered}
$$

\section{B. Subequations of degree 3}

Denoting $\omega_{k} k=1,2,3$, cubic roots of unity, each such subequation has the necessary form

$$
\begin{aligned}
F\left(u, u^{\prime}\right) \equiv & -\left(\omega_{1} a u^{\prime}+u^{2}\right)\left(\omega_{2} a u^{\prime}+u^{2}\right)\left(\omega_{3} a u^{\prime}+u^{2}\right)+b_{1} u^{\prime 2} u+b_{2} u^{\prime} u^{3}+b_{3} u^{5}+b_{4} u^{\prime 2}+b_{5} u^{\prime} u^{2} \\
& +b_{6} u^{4}+b_{7} u^{\prime} u+b_{8} u^{3}+b_{9} u^{\prime}+b_{b} u^{2}+b_{a} u+b_{c}+b_{0}=0
\end{aligned}
$$

with all $\omega_{j}$ distinct and the additional condition to be irreducible.

The first order third degree subequation is precisely defined as

$$
\begin{aligned}
F\left(u, u^{\prime}\right) \equiv & -a^{3} u^{\prime 3}-u^{6}+b_{1} u^{\prime 2} u+b_{2} u^{\prime} u^{3}+b_{3} u^{5}+b_{4} u^{\prime 2}+b_{5} u^{\prime} u^{2}+b_{6} u^{4}+b_{7} u^{\prime} u+b_{8} u^{3}+b_{9} u^{\prime} \\
& +b_{b} u^{2}+b_{a} u+b_{c}+b_{0}=0 .
\end{aligned}
$$

The algorithm ${ }^{13}$ to compute the coefficients $b_{k}$ is to substitute $u$ by one of the Laurent series (4), which makes the right hand side of (19) become a Laurent series

$$
F\left(u, u^{\prime}\right) \equiv \sum_{j=0}^{+\infty} F_{j}\left(z-z_{0}\right)^{j-6}
$$

then to solve the infinite set of equations

$$
\forall a \forall j: F_{j}=0 .
$$

The practical resolution is as follows. First, the 21 equations $F_{j}=0, j=0, \ldots, 6$, define a linear system for the $b_{k}$, which admits a unique solution and generates six nonlinear constraints among the six $c_{k}$. By considering slightly more equations in (21) (in the present case, going to $j=8$ is enough), the set of nonlinear constraints among the $c_{k}$ 's admits exactly two solutions, and all the remaining equations $F_{j}=0$ identically vanish. These two solutions are 


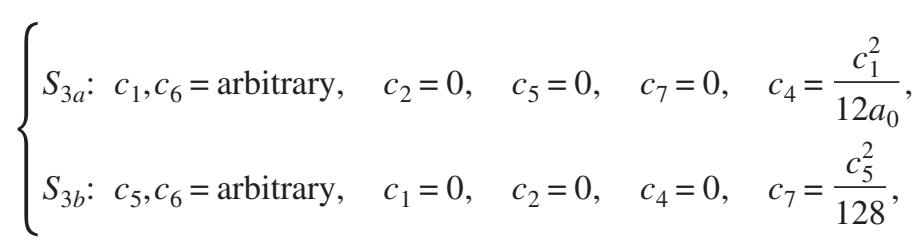

and they are identical to the two residue conditions (17) and (15).

The corresponding subequations have genus one

$$
\begin{gathered}
\left(a u^{\prime}+4 k_{1} u\right)^{2}\left(a u^{\prime}-2 k_{1} u\right)+\left(u^{3}+20 k_{1}^{3}+k_{6}\right)^{2}=0, \quad c_{1}=12 a^{2} k_{1}, \quad c_{6}=4 k_{6}, \\
\left(a u^{\prime}\right)^{3}+\left(u^{3}-3 k_{5}^{2} u+k_{6}\right)^{2}=0, \quad c_{5}=-16 k_{5}^{2}, \quad c_{6}=4 k_{6} .
\end{gathered}
$$

The method to integrate them (Ref. 1, Sec. 249, p. 393) is to build a birational transformation to the canonical equation of Weierstrass,

$$
\wp^{\prime 2}=4\left(\wp-e_{1}\right)\left(\wp-e_{2}\right)\left(\wp-e_{3}\right)=4 \wp^{3}-g_{2} \wp-g_{3} .
$$

To do that, it proves convenient to introduce one of the roots $e_{0}$ of the cubic polynomial of $u(x)$ appearing as a square in (23) and (24), i.e., to redefine $k_{6}$ by the respective relations

$$
e_{0}^{3}+20 k_{1}^{3}+k_{6}=0 \text { and } e_{0}^{3}-3 k_{5}^{2} e_{0}+k_{6}=0 .
$$

Subequation (24) is one of the five first order binomial equations of Briot and Bouquet (Ref. 2 , p. 105), its general solution is classical

$$
\begin{gathered}
\frac{1}{u-e_{0}}=\frac{\wp^{\prime}\left(z-z_{0}, g_{2}, g_{3}\right)-A}{N_{1}}, \quad g_{2}=0, \quad g_{3}=\frac{\left(e_{0}^{2}-k_{5}^{2}\right)^{2}\left(e_{0}^{2}-4 k_{5}^{2}\right)}{243 a^{6}}, \\
N_{1}=\frac{2\left(e_{0}^{2}-k_{5}^{2}\right)^{2}}{3 a^{3}}, \quad A=\frac{e_{0}\left(e_{0}^{2}-k_{5}^{2}\right)}{3 a^{3}} .
\end{gathered}
$$

Subequation (23) has been integrated by Briot and Bouquet (Ref. 1, Sec. 250, p. 395) by introducing a function $w$ defined by

$$
a u^{\prime}+4 k_{1} u=\frac{u^{3}-e_{0}^{3}}{u-e_{0}} w,
$$

then by establishing the birational transformation

$$
w=\frac{a u^{\prime}+4 k_{1} u}{u^{2}+e_{0} u+e_{0}^{2}}, \quad u=\frac{-3 a w w^{\prime}-e_{0} w^{3}+6 k_{1} w^{2}+2 e_{0}}{2\left(w^{3}+1\right)},
$$

finally by integrating the ODE for $w$,

$$
\begin{gathered}
w=\frac{2 k_{1}}{e_{0}}+\frac{A}{\wp-B}, \quad g_{2}=\frac{4 k_{1}\left(k_{1}^{3}-e_{0}^{3}\right)}{3 a^{4}}, \quad g_{3}=\frac{e_{0}^{6}-20 e_{0}^{3} k_{1}^{3}-8 k_{1}^{6}}{17 a^{6}}, \\
g_{2}^{3}-27 g_{3}^{2}=-\frac{\left(8 k_{1}^{3}+e_{0}^{3}\right)^{3} e_{0}^{3}}{27 a^{12}}, \quad A=-\frac{e_{0}^{3}+8 k_{1}^{3}}{3 a^{2}}, \quad B=-\frac{k_{1}^{2}}{a^{2}} .
\end{gathered}
$$

More generally, birational transformations from $\left(u, u^{\prime}\right)$ to $\left(\wp, \wp^{\prime}\right)$ are obtained with an algorithm due to Poincaré, implemented, for instance, by the command Weierstrassform of the computer algebra package algcurves. ${ }^{11}$ 


\section{Subequations of degree 2}

Let us define the second degree subequation as

$$
F\left(u, u^{\prime}\right) \equiv a^{2} u^{\prime 2}-a u^{2} u^{\prime}+u^{4}+b_{4} u^{\prime} u+b_{3} u^{3}+b_{5} u^{\prime}+b_{2} u^{2}+b_{1} u+b_{0}=0,
$$

with the additional condition to be irreducible. Computations similar to those mentioned in Sec. II B provide two solutions,

$$
\left\{\begin{array}{l}
S_{2 A}: \quad c_{1}, c_{4}=\text { arbitrary, } \quad c_{2}=0, \quad c_{5}=\frac{c_{1}^{2}-12 a^{3} c_{4}}{4 a^{4}} \\
c_{6}=-\frac{c_{1}\left(c_{1}^{2}+36 a^{3} c_{4}\right)}{144 a^{6}} \\
c_{7}=\frac{\left(12 a^{3} c_{4}-c_{1}^{2}\right)\left(36 a^{3} c_{4}-11 c_{1}^{2}\right)}{1536 a^{8}} \\
u=v-\frac{k_{1}}{2}, \quad c_{1}=-3 a^{2} k_{1}, \quad c_{4}=2 a b^{2}+\frac{3}{4} a k_{1}^{2} \\
\left(a v^{\prime}-\frac{v^{2}-b^{2}}{2}\right)^{2}+\frac{3}{4}(v+b)(v-b)\left(v-k_{1}\right)^{2}=0, \quad b \neq 0
\end{array}\right.
$$

and

$$
\left\{\begin{array}{l}
S_{2 B}: c_{1}, c_{2}=\text { arbitrary, } \quad c_{4}=\frac{44 c_{1}^{2}+8 a c_{1} c_{2}-a^{2} c_{2}^{2}}{144 a^{3}} \\
c_{5}=\frac{-32 c_{1}^{2}-24 a c_{1} c_{2}-7 a^{2} c_{2}^{2}}{48 a^{4}} \\
c_{6}=-\frac{\left(c_{1}+a c_{2}\right)\left(12 c_{1}^{2}+6 a c_{1} c_{2}+a^{2} c_{2}^{2}\right)}{144 a^{6}} \\
c_{7}=-\frac{\left(4 c_{1}+3 a c_{2}\right)\left(48 c_{1}^{2}+20 a c_{1} c_{2}+a^{2} c_{2}^{2}\right)}{55296 a^{7}} \\
u=v+\frac{b}{4}+\frac{c_{1}}{12 a^{2}}, \quad c_{2}=-2 \frac{c_{1}}{a}+6 a b \\
\left(a v^{\prime}-\frac{v^{2}-b^{2}}{2}\right)^{2}+\frac{3}{4}(v+b)^{3}(v-b)=0, \quad b \neq 0 .
\end{array}\right.
$$

For $k_{1}^{2} \neq b^{2}$, the point transformation

$$
v=k_{1}+\frac{1}{w}, \quad w=-\frac{1}{k_{1}+b}-\frac{1}{k_{1}-b}+N\left(\lambda-\frac{1}{\lambda}\right), \quad N^{2}=-\frac{b^{2}}{\left(k_{1}^{2}-b^{2}\right)^{2}},
$$

maps the ODE (32) to the Riccati ODE

$$
a N \lambda^{\prime}-M \lambda-\frac{b^{2}}{4\left(k_{1}^{2}-b^{2}\right)}\left(\lambda^{2}+1\right)=0, \quad M^{2}=\frac{3 b^{2}}{4\left(k_{1}^{2}-b^{2}\right)},
$$

whose general solution is a Möbius function of one exponential so that $v$ is a rational function of one exponential.

For $k_{1}^{2}=b^{2}$, i.e., for instance, for $k_{1}=-b$, the ODE (33) integrates as

$$
v=-b+\frac{2 b}{w}, \quad w=1+3\left(1+e^{b\left(z-z_{0}\right) /(2 a)}\right)^{2} .
$$




\section{Subequations of degree 1}

These first degree subequations

$$
F\left(u, u^{\prime}\right) \equiv a u^{\prime}+u^{2}+b_{1} u+b_{0}=0
$$

are determined by requiring their vanishing when $u$ is the Laurent series (4). This results in

$$
\left\{\begin{aligned}
& S_{1}: c_{1}, c_{2}, c_{4}, c_{5}=\text { arbitrary } \\
& b_{1}=\frac{2 c_{1}-a c_{2}}{12 a^{2}} \\
& b_{0}=\frac{44 c_{1}^{2}-32 a c_{1} c_{2}+5 a^{2} c_{2}^{2}-144 a^{3} c_{4}+144 a^{4} c_{5}}{1152 a^{4}} \\
& 1152 a^{6} c_{6}=-56 c_{1}^{3}+60 a c_{1}^{2} c_{2}-18 a^{2} c_{1} c_{2}^{2}+a^{3} c_{2}^{3}+288 a^{3} c_{1} c_{4} \\
&-144 a^{4} c_{2} c_{4}-96 a^{4} c_{1} c_{5}+48 a^{5} c_{2} c_{5} \\
& 2^{13} 3^{2} a^{8} c_{7}=-176 c_{1}^{4}+128 a c_{1}^{3} c_{2}+24 a^{2} c_{1}^{2} c_{2}^{2}-32 a^{3} c_{1} c_{2}^{3} \\
&+5 a^{4} c_{2}^{4}+2688 a^{3} c_{1}^{2} c_{4}-1536 a^{4} c_{1} c_{2} c_{4}+96 a^{5} c_{2}^{2} c_{4} \\
&-6912 a^{6} c_{4}^{2}+128 a^{4} c_{1}^{2} c_{5}-512 a^{5} c_{1} c_{2} c_{5}+224 a^{6} c_{2}^{2} c_{5} \\
&+4608 a^{7} c_{4} c_{5}+2304 a^{8} c_{5}^{2} .
\end{aligned}\right.
$$

The solution of this Riccati equation is either a rational function of one exponential or a rational function,

$$
u=\left\{\begin{array}{l}
-\frac{b_{1}}{2}+a \frac{k}{2} \operatorname{coth} \frac{k}{2}\left(z-z_{0}\right), \quad k^{2}=\frac{b_{1}^{2}-4 b_{0}}{2 a^{2}} \neq 0 \\
-\frac{b_{1}}{2}+\frac{a}{z-z_{0}}, \quad b_{1}^{2}-4 b_{0}=0 .
\end{array}\right.
$$

\section{ACKNOWLEDGMENTS}

The authors would like to thank the referee for the valuable suggestions. R.C. and T.-W.N. were partially supported by PROCORE-France/Hong Kong Joint Research under Grant No. F-HK29/05T and RGC under Grant No. HKU 703807P.

${ }^{1}$ Briot, C. and Bouquet, J.-C., Théorie des fonctions elliptiques, 1st ed. (Mallet-Bachelier, Paris, 1859); Théorie des fonctions elliptiques, 2nd ed. (Gauthier-Villars, Paris, 1875).

${ }^{2}$ Conte, R., "The Painlevé approach to nonlinear ordinary differential equations," The Painlevé Property, One Century

Later, CRM Series in Mathematical Physics, edited by R. Conte (Springer, New York, 1999), pp. 77-180.

${ }^{3}$ Conte, R. and Musette, M., "Elliptic general analytic solutions," Stud. Appl. Math. 123, 63 (2009).

${ }^{4}$ Eremenko, A. E., "Meromorphic solutions of equations of Briot-Bouquet type," Teor. Funktsii, Funktsional'nyi Analiz i Prilozhen, Vyp. 16, 48 (1982) [Am. Math. Soc. Transl. 133, 15 (1986)].

${ }^{5}$ Eremenko, A. E., "Meromorphic traveling wave solutions of the Kuramoto-Sivashinsky equation," Math. Phys., Anal. Geom. 2, 278 (2006).

${ }^{6}$ Eremenko, A. E., Liao, L. W., and Ng, T. W., "Meromorphic solutions of higher order Briot-Bouquet differential equations," Math. Proc. Cambridge Philos. Soc. 146, 197 (2009).

${ }^{7}$ Halphen, G.-H., Traité des fonctions elliptiques et de leurs applications (Gauthier-Villars, Paris, 1886), Vol. 1; Traité des fonctions elliptiques et de leurs applications (Gauthier-Villars, Paris, 1888), Vol. 2; Traité des fonctions elliptiques et de leurs applications (Gauthier-Villars, Paris, 1891), Vol. 3.

${ }^{8}$ Hayman, W. K., Meromorphic Functions, Oxford Mathematical Monographs (Clarendon, Oxford, 1964).

${ }^{9}$ Hille, E., Ordinary Differential Equations in the Complex Domain (Wiley, New York, 1976).

${ }^{10}$ Hone, A. N. W., "Non-existence of elliptic travelling wave solutions of the complex Ginzburg-Landau equation," Physica D 205, 292-306 (2005).

${ }^{11}$ van Hoeij, M., package "algcurves," MAPLE v (1997), http://www.math.fsu.edu/hoeij/algcurves.html.

${ }^{12}$ Laine, I., Nevanlinna Theory and Complex Differential Equations (de Gruyter, Berlin, 1992).

${ }^{13}$ Musette, M. and Conte, R., "Analytic solitary waves of nonintegrable equations," Physica D 181, 70 (2003).

${ }^{14}$ Nevanlinna, R., Le théorème de Picard-Borel et la théorie des fonctions méromorphes (Gauthier-Villars, Paris, 1929).

${ }^{15}$ Painlevé, P., "Mémoire sur les équations différentielles dont l'intégrale générale est uniforme," Bull. Soc. Math. France 
28, 201 (1900).

${ }^{16}$ van Saarloos, W., "Front propagation into unstable states," Phys. Rep. 386, 29 (2003).

${ }^{17}$ Yang, C. C. and Ye, Z., "Estimates of the proximity function of differential polynomials," Proc. Jpn. Acad., Ser. A: Math. Sci. 83, 50 (2007) 
Journal of Mathematical Physics is copyrighted by the American Institute of Physics (AIP). Redistribution of journal material is subject to the AIP online journal license and/or AIP copyright. For more information, see http://ojps.aip.org/jmp/jmpcr.jsp 